\title{
The Correlation between Beck Depression Inventory II (BDI II) Score with Serum Magnesium Levels in Patients on Regular Hemodialysis
}

\author{
Miftahul Ihsan ${ }^{1}$, Wika Hanida Lubis ${ }^{2}$, Alwi Thamrin Nasution² \\ ${ }^{1}$ Resident, Department of Internal Medicine, Faculty of Medicine, Universitas Sumatera Utara, Medan 20155, \\ Indonesia \\ ${ }^{2}$ Department of Internal Medicine, Faculty of Medicine, Universitas Sumatera Utara, Medan, 20155, Indonesia
}

Corresponding Author: Miftahul Ihsan

\begin{abstract}
Objective: To assess the correlation between BDI II score as screening tools for early symptoms of depression with serum magnesium levels in regular hemodialysis patients.

Methods: A cross-sectional study conducted on 48 patients with chronic kidney disease on regular HD in RSUP Haji Adam Malik. Patients included in this study were $\geq 18$ years of age, on regular HD with a duration of $\geq 3$ months, and were willing to be included in the study by signing a consent form after explanation. Patients with other psychiatric comorbidities other than depression and patients taking medicine that affect serum magnesium levels were excluded from the study. All studied patients were assessed for serum magnesium levels and depression symptoms using the BDI II questionnaire.
\end{abstract}

Results: In this study we found that there is a significant correlation between serum Magnesium levels and BDI II score with a weak correlation strength level $(r=-0.35)$. The BDI II score in regular hemodialysis patients was found to be in a score of 4-15 with a median score of 7. Magnesium levels in regular hemodialysis patients ranged from 1.78 to 3.75 $\mathrm{mg} / \mathrm{dl}$, with a median of 2.9.

Conclusions: In this study, there is a significant correlation found between serum Magnesium levels and BDI II score in patient on regular HD.

Keywords: Beck Depression Inventory II (BD

II) score, Chronic Kidney Disease,

Hemodialysis, Serum magnesium levels.

\section{INTRODUCTION}

Depression is a psychological disorder that is often found in patients with chronic diseases, including in patients with chronic kidney disease (CKD). Several studies have shown that moderate to severe depressive symptoms occur in $25-50 \%$ of CKD patients receiving hemodialysis therapy. It has been proven that there is a significant relationship between depression and mortality in regular hemodialysis patients. ${ }^{[1]}$

Depression is highly associated with various chronic diseases, including chronic kidney disease. Depression in CKD patients undergoing hemodialysis can increase the mortality rate regardless of the regularity of hemodialysis itself. The hospitalization rate for CKD patients with mental disorders is 1.5 - 3.0 times higher than that of patients with other chronic diseases. It is also said that depression is an independent risk factor for mortality in CKD patients. ${ }^{[1]}$

The prevalence of depression in CKD patients has not been determined with certainty. Various studies have identified the magnitude of the incidence of depression in patients with CKD as high as $38 \%-46 \%$. In Indonesia alone, data from Cipto Mangunkusumo Hospital Jakarta found that the prevalence of depression in CKD patients undergoing hemodialysis reached $31.1 \%$. The occurrence of depression in CKD patients has a direct impact on 
worsening the prognosis of the patient's disease, increasing noncompliance with drug consumption, increasing the number of negligence in hemodialysis visits, reducing quality of life and ultimately increasing mortality. ${ }^{[2]}$

CKD is associated with a state of systemic inflammation and increased levels of urea in the blood (uremia) which are thought to have a positive correlation with the incidence of depression. Psychosocial stressors experienced by CKD patients also exacerbate the depressive symptoms that some CKD patients may experience. ${ }^{[3]}$ In recent years, many studies have started to try to identify the effect of electrolyte components on the incidence of depression in CKD patients undergoing hemodialysis, one of which is magnesium. ${ }^{[4]}$

To screen for depression in hemodialysis patients, a Beck Depression Inventory II (BDI-II) examination consisting of 21 questions can be performed. The higher the BDI II score, the more severe the symptoms of depression. Screening by distributing BDI II questionnaires to patients, and filled in within 15 minutes. If the results of the questionnaire get a score of $\geq 14$, it is possible that depression may occur. Furthermore, a definite diagnosis is carried out based on the Diagnostic and Statistical Manual and Mental Disorder-V (DSM-V). ${ }^{[5]}$

Magnesium is a cation capable of modulating the activity of N-methyl-Daspartate (NMDA) and GammaAminobutyric acid (GABA) receptors. Low serum magnesium levels will result in large amounts of calcium and sodium entering post-synaptic neurons, along with the occurrence of potassium efflux. The entry of large amounts of calcium will result in the formation of reactive oxygen species (ROS) which are toxic to cells and increase the risk of depression. ${ }^{[6]}$

In regular hemodialysis patients, serum magnesium depends on the concentration of the hemodialysis solution. With a decrease in renal function, the excretory capacity for magnesium decreases significantly. Several cohort studies suggest that hypomagnesemia can be used as a predictor of mortality and decreased function in CKD patients as much as mortality in hemodialysis patients. All of these exposures, it is confirmed that abnormalities of magnesium level occur in patients with CKD. ${ }^{[6]}$

Sarefko et al. proved that magnesium levels are inversely related to the severity of depression as measured by the Hamilton score where the lower the serum magnesium level, the more severe depression experienced by the patient. Similar study conducted by Nechifor et al. also found that low magnesium levels are associated with more severe depression symptoms. ${ }^{[7]}$ However, several other studies actually found conflicting results. A study conducted by Widmer et al. and Imada et al. found that magnesium levels were higher in people with depression. ${ }^{[8-9]}$

The variety of study results related to the relationship between magnesium and depression made researchers interested in conducting a study that identified a correlation between BDI II score as a screening for early symptoms of depression with magnesium levels in blood in regular hemodialysis patients, especially at RSUP H Adam Malik, Medan.

\section{MATERIAL AND METHODS 2.1. Study design}

This study is an analytical study with a cross-sectional design which aims to determine the correlation between BDI II score and serum magnesium levels in regular hemodialysis patients.

\subsection{Study participants}

The target population in this study were regular hemodialysis patients in Medan City, while the accessible population was regular hemodialysis patients at RSUP HAM Medan in 2020.

The sampling technique used in this study was consecutive sampling, in which all subjects were involved in the study as 
long as they met the inclusion and exclusion criteria.

\section{Inclusion Criteria:}

a. Male and female patients $\geq 18$ years old

b. Chronic kidney disease patients undergoing hemodialysis therapy with a duration of $\geq 3$ months

c. Willing to be included in the research by signing the consent form after the explanation

\section{Exclusion Criteria:}

a. Patients with other psychiatric comorbidities other than depression

b. Patients taking medications that affect serum Magnesium levels

The minimum sample size required is calculated based on the sample formula to test the hypothesis of unpaired numerical data, is 48 patients.

\subsection{Procedure}

Every patient diagnosed with chronic kidney disease at RSUP Haji Adam Malik Medan who meets the inclusion criteria and does not meet the exclusion criteria is the subject of the study and the patient will fill out a letter of consent after receiving an explanation. The research samples were selected consecutively. The subject of the study was carried out with venous blood sampling through the median cubital vein for examination of kidney function and measurement of magnesium levels in the blood. Examination of magnesium levels in the blood was carried out using spectrophotometric techniques. All study subjects were assessed for depression symptoms using the BDI II questionnaire. Statistical analysis of research data was carried out.

\subsection{Measurements}

\subsubsection{BDI II score}

The total score obtained by the patient after filling out the BDI II questionnaire.

\subsubsection{Serum magnesium level}

The intracellular divalent cation of $\mathrm{Mg}^{2+}$ which is measured using the Spectrophotometric technique in $\mathrm{mEq} / \mathrm{L}$.

\subsubsection{Chronic kidney disease}

Structural or functional abnormalities in the kidneys that last more than three months which is measured by the glomerular filtration rate from blood work.

\subsubsection{Regular hemodialysis}

Patients undergoing hemodialysis for more than 3 months which is assessed from medical report.

\subsection{Statistical Methods}

Univariate, bivariate and multivariate analyzes were performed on the data in this study. Univariate analysis was performed to obtain the distribution of sample characteristics.

Bivariate analysis was performed to determine the relationship between the independent and dependent variables. Before the bivariate test was carried out, the data normality test was conducted first with the Kolmogorov Smirnov test. To determine the correlation between BDI II scores and magnesium levels in regular hemodialysis patients, a Pearson Correlation test was performed if the data were normally distributed, and the Spearman Correlation test if the data were not normally distributed. Statistical analysis was performed using the SPSS 20 (Statistical Package for Social Sciences) computer program where the $\mathrm{p}$ value $<0.05$ was significant.

\subsection{Ethical clearance}

Ethical clearance is a written statement provided by the research ethics commission for research involving living things as well as humans, animals and plants, where it is stated that a research proposal is feasible after meeting certain requirements. This research will be conducted after obtaining permission to carry out research from the research supervisor, approval from the USU Medical Faculty Research Ethics Commission, and 
after obtaining permission from the head of the hemodialysis unit at RSUP HAM.

As ethical considerations, researchers believe that respondents' rights is secured by paying attention to aspects such as Self Determination, Informed Consent, Privacy, Anonymity and Confidentiality, and Protection from Discomfort.

\section{RESULTS}

\subsection{Sample characteristics}

Table 1 shows that the patients who were the subjects in this study were almost equal between the sexes of men and women. Of the 48 subjects, 25 were male and 23 were female. In terms of age, most of the study subjects were in the 40-59 years age group, namely as many as $47.9 \%$ of subjects, followed by patients who were over or equal to 60 years (as many as $27.1 \%$ of cases). Patients aged less than 40 years amounted to only $25 \%$ of all study subjects.

Based on the disease that underlies the occurrence of chronic renal failure, it was found that the main cause of CKD in patients was hypertension which reached $66.7 \%$ or about $2 / 3$ of the total patients who were the subjects of this study. The second cause that became the most common cause of CKD in this study was Type 2 Diabetes Mellitus, and the combination of Type 2 Diabetes Mellitus + Hypertension, which each amounted to $10.4 \%$ of the study subjects. A total of 4 subjects experienced urolithiasis as the cause of CKD. There were 2 subjects who experienced CKD due to other reasons, namely nephrotic syndrome (1 subject) and autoimmune (1 subject).

Table 1. Baseline characteristics

\begin{tabular}{|c|c|c|c|}
\hline & Variable & $\mathbf{n}$ & $\%$ \\
\hline \multirow[t]{2}{*}{ Gender } & Male & 25 & 52.1 \\
\hline & Female & 23 & 47.9 \\
\hline \multirow{3}{*}{$\begin{array}{l}\text { Age in } \\
\text { years }\end{array}$} & $<40$ & 12 & 25.0 \\
\hline & $40-59$ & 23 & 47.9 \\
\hline & $\geq 60$ & 13 & 27.1 \\
\hline \multirow{6}{*}{$\begin{array}{l}\text { Underlying } \\
\text { disease }\end{array}$} & Urolithiasis & 4 & 4.2 \\
\hline & Type 2 Diabetes Mellitus & 5 & 10.4 \\
\hline & $\begin{array}{l}\text { Type } 2 \text { Diabetes } \text { Mellitus }+ \\
\text { Hypertension }\end{array}$ & 5 & 10.4 \\
\hline & Hypertension & 32 & 66.7 \\
\hline & Others & 2 & 2.1 \\
\hline & TOTAL & 48 & 100.0 \\
\hline
\end{tabular}

\subsection{Correlation between Magnesium Levels with BDI Score}

All data obtained were tested for normality using the Shapiro Wilk test, where it was found that the data on Magnesium levels were normally distributed, but the BDI II score data were not normally distributed. Therefore, to determine the correlation between BDI II score and serum magnesium levels in regular hemodialysis patients, the Spearman Correlation test was performed, with the following results:

Table 2. Correlation between Magnesium Levels with BDI Score

\begin{tabular}{|l|l|l|l|l|}
\hline Variable & mean \pm SD & $\begin{array}{l}\text { median } \\
(\mathbf{m i n}-\mathbf{m a x})\end{array}$ & $\mathbf{r}$ & $\begin{array}{l}\text { p- } \\
\text { value }\end{array}$ \\
\cline { 1 - 3 } $\begin{array}{l}\text { Serum } \\
\text { magnesium } \\
\text { level }\end{array}$ & $2.8 \pm 0.46$ & $\begin{array}{l}2.9 \\
(1.78-3.75)\end{array}$ & -0.35 & $0.014^{*}$ \\
\cline { 1 - 2 } & $7.5 \pm 2.90$ & $7.0(4-15)$ & \\
\hline \multicolumn{2}{|l|}{ *) significant with the Spearman Correlation test }
\end{tabular}

Table 2 shows that the mean serum magnesium level in chronic renal disease patients on regular hemodialysis is $2.8 \pm$ $0.46 \mathrm{mEq} / \mathrm{L}$, which means that it is still within the normal range of magnesium levels. As for the BDI II score, it was found that the mean BDI II score of the patient who was the subject of the study was 7 , with the lowest score being 4 and the highest score being 15 .

The results of statistical analysis using the Spearman Correlation test showed that there was a significant correlation between magnesium levels and BDI II score ( $p<0.05$ ), with a weak correlation strength level $(r=-0.35)$. The negative correlation coefficient value indicates that there is an opposite relationship between magnesium levels and BDI II score. In other words, the higher a patient's Magnesium level, the lower his BDI II score. Conversely, the lower the patient's Magnesium level, the higher the patient's BDI II score.

To clarify the correlation between serum Magnesium levels and BDI II scores in patients on regular hemodialysis, the correlation is described in the form of a scatter plot, as follows: 


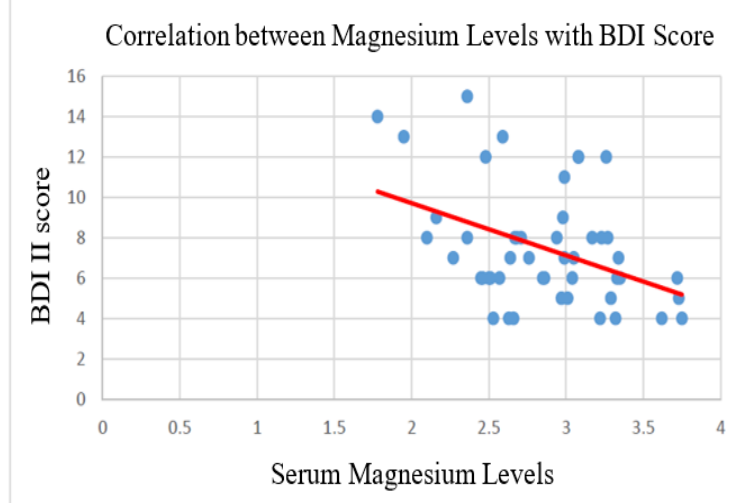

Fig. 1. Scatter diagram of Correlation between Magnesium level with BDI score

\subsection{BDI II Score in Patients with Regular HD}

Further analysis was carried out to determine the factors associated with the severity of depression in patients on regular hemodialysis through the BDI II score instrument. Therefore, the mean difference test was carried out using the Kruskal Wallis test with the following results:

\begin{tabular}{|c|c|c|c|c|}
\hline & Variable & Median & Min-Max & p value \\
\hline \multirow[t]{2}{*}{ Gender } & Male & 7 & $4-15$ & \multirow[t]{2}{*}{0.62} \\
\hline & Female & 7 & $4-14$ & \\
\hline \multirow[t]{3}{*}{ Age in years } & $<40$ & 5 & $4-6$ & \multirow[t]{3}{*}{$<0.01 *$} \\
\hline & $40-59$ & 7 & $4-11$ & \\
\hline & $\geq 60$ & 12 & $7-15$ & \\
\hline \multirow[t]{5}{*}{ Underlying disease } & Urolithiasis & 10 & $6-13$ & \multirow[t]{5}{*}{$<0.02 *$} \\
\hline & Type 2 Diabetes Mellitus & 8 & $4-12$ & \\
\hline & Type 2 Diabetes Mellitus + Hypertension & 9 & $8-15$ & \\
\hline & Hypertension & 6 & $4-14$ & \\
\hline & Others & 8 & $6-11$ & \\
\hline
\end{tabular}

Table 3 shows that there is a significant relationship between age and BDI II score. Regular hemodialysis patients who were in the age group over or equal to 60 years had the highest BDI II score when compared to other age groups $(p<0.05)$. In contrast, patients younger than 40 years had the lowest BDI II score. Therefore, it can be interpreted that the BDI II score is directly proportional to age, where the older the age of a patient undergoing regular hemodialysis, the heavier the degree of depression experienced by the patient.

Furthermore, it is also known that the diagnosis of the underlying disease has a significant relationship with the incidence of depression ( $p$ <.05). Patients with an underlying disease of urolithiasis had the highest BDI score compared to other underlying diseases. However, even though the BDI-II score in the urolithiasis patient was higher than in the other disease groups, these scores were also in the mild depression range (score 0-13).

The analysis based on gender groups showed that there was no significant relationship between gender and the severity of depression in patients undergoing regular hemodialysis ( $p>0.05)$.

\section{DISCUSSION}

This study found that clinical symptoms of depression were found in almost all CKD patients on regular dialysis with varying degrees of severity as measured by the BDI II score. The lowest score in the subjects of this study was 4 , and the highest score was 15. These data indicate that the spectrum of clinical manifestations of depression can vary widely between individual from mild to moderate. Therefore, it is necessary to analyze the factors that are thought to have contributed to the severity of depression symptoms in CKD patients undergoing hemodialysis.

One of the factors that is thought to have contributed to the severity of depression symptoms in patients undergoing hemodialysis is serum magnesium levels. This study proves that there is a significant negative correlation between serum 
magnesium levels and depression symptom severity as measured by BDI II score ( $p$ $<0.05)$. Statistical analysis shows that the correlation coefficient is $\mathrm{r}=-0.35$, which means that there is a negative correlation which is weak. This value means that the higher the serum Magnesium level, the lower or lighter the depression symptoms experienced by the patient. Conversely, the lower the serum Magnesium level, the higher or heavier the depression symptoms the patient exhibits.

The findings of this study are in line with the results of research by Nechifor et al. which showed decreased erythrocyte magnesium levels in severe and moderate major depression patients. Another study also observed that erythrocyte magnesium levels were lower (compared to a control group) in adult patients with major depression who had received antidepressant therapy prior to hospital admission. Another study that also identified an association between serum magnesium levels and depression scores using the Hamilton questionnaire also found a negative correlation between magnesium levels and Hamilton score, as well as the results of this study. ${ }^{[7]}$

Depression is defined as a state of disruption of human function related to feelings of sadness and accompanying symptoms, including changes in sleep and appetite patterns, psychomotor, concentration, anhedonia, fatigue, feelings of hopelessness and helplessness, and suicidal thoughts. Clinical manifestation of depression syndrome should consist of characteristic symptoms including anhedonia and feelings of sadness, uselessness, guilt, hopelessness, etc. and followed by disturbances in sleep, appetite, and libido. ${ }^{[10]}$

Depression is a psychological disorder that is often found in patients with chronic diseases, including in patients with chronic kidney disease (CKD). Depression in CKD patients on dialysis can increase the mortality rate regardless of their regular dialysis routine. The hospitalization rate for
CKD patients with mental disorders is 1.5 3.0 times higher than that of patients with other chronic diseases. It is also said that depression is an independent risk factor for mortality in CKD patients. ${ }^{[1]}$

The incidence of depression in patients with CKD is reported to be very diverse. In general, it is estimated that depression occurs in at least one third of patients with CKD on dialysis. Several studies have shown that moderate to severe depressive symptoms occur in $25-50 \%$ of CKD patients receiving dialysis therapy. A study conducted by Smith et al. found that the incidence of depression in patients with CKD was $47 \%$. This is not much different from the findings of Wuerth et al, who found that the incidence of depression was $49 \%$ in patients with CKD. In Indonesia alone, data from Cipto Mangunkusumo Hospital Jakarta found that the prevalence of depression in CKD patients undergoing hemodialysis reached $31.1 \%$. The occurrence of depression in CKD patients has a direct impact on worsening the prognosis of the patient's disease, increasing noncompliance with drug consumption, increasing the number of negligence in dialysis visits, reducing quality of life and ultimately increasing mortality. ${ }^{[2]}$

Beck Depression Inventory (BDI), created in 1961 by Dr. Aaron T. Beck, and was developed to assess the behavioral manifestations of depression in adolescents and adults. Designed to standardize an assessment of depression severity and simply describe depressive symptoms. The BDI is the most widely used instrument for assessing the severity of depression. The higher the total score indicates the more severe depressive symptoms. ${ }^{[5]}$

Magnesium is a cation capable of modulating the activity of NMDA and GABA receptors. Low serum magnesium levels will result in large amounts of calcium and sodium entering the postsynaptic neurons, coinciding with the occurrence of potassium efflux. The entry of large amounts of calcium will result in the formation of reactive oxygen species (ROS) 
which are toxic to cells and increase the risk of depression. ${ }^{[6]}$

However, there are still some differences regarding this finding with the results of other studies. There is evidence of increased erythrocyte magnesium levels in people experiencing major and moderate depression versus healthy individuals. A positive correlation between erythrocyte magnesium concentration and the development of clinical symptoms of depression has also been reported by several studies. Therefore, plasma/serum magnesium levels are unlikely to be a reliable indicator of depressive disorders, as ambiguous results have been found several times. Eby et al. proposed the hypothesis that tissue magnesium levels are a much better indicator of plasma /serum magnesium concentrations. Iosifescu et al. and Nowak et al. reported an association between low brain magnesium concentrations and depression. ${ }^{[11]}$

The variety of studies results related to the relationship between magnesium and depression has made magnesium not used as a routine biomarker for predictors of depression in people with CKD.

The incidence of depression in patients with CKD on regular hemodialysis is not only influenced by serum magnesium levels. Psychosocial stressors experienced by CKD sufferers also exacerbate the depressive symptoms that some CKD sufferers may experience.

CKD can be classified as a stressor, which is an event that causes stress to a person. Patients undergoing hemodialysis therapy generally experience stress. CKD patients on hemodialysis therapy think that in order to survive they will always have to depend on dialysis machines. This often creates thoughts in the patient that his life will be threatened and that hope for life will be reduced. Patients experience fear that their age will not be long, and this problem also creates conflict in the family.

The life expectancy of patients with CKD has indeed increased in recent decades with advances in dialysis techniques.
However, it turns out that dialysis does not fully restore the patient's quality of life to normal. The decline in quality of life was evident in the group of patients who had been on HD for a long time. This group of patients complained about many problems related to opportunities for activity, expenses incurred, the burden of limiting fluid consumption, and even services provided by health workers. All these aspects cause CKD patients, especially those undergoing dialysis, often fall into a state of depression. ${ }^{[4]}$

The analysis was continued by looking at whether there was a relationship between age and the severity of depression symptoms experienced by CKD patients on regular HD. This study shows that there is a significant relationship ( $p<0.001)$ between age and the severity of depression symptoms. Patients in the age group above or equal to 60 years have the highest symptoms of depression when compared to other age groups. Patients over 60 years of age had a median BDI II score of 12 , with the lowest score of 7 and the highest score of 15. Lower depression scores were found in the 40-59 year age group with the median score of 7 . In contrast, BDI II scores the lowest was found in the $<40$ years age group, with the median value being 5 .

The 2018 Ministry of Health's Basic Health Research notes that the prevalence rate of depression in Indonesia in the elderly reaches $23.3 \%$, and this will increase in the elderly with chronic diseases. Based on data compiled by the CDC in 2007-2010, the highest prevalence of depression suffered by the 40-59 year old group, was $9.45 \%$.

The results of the 2018 Basic Health Research stated that the incidence of depression tends to be experienced by men $(30.6 \%)$, the age group $\geq 70$ years $(30.6 \%)$, low education level $(24.4 \%)$, unoccupational (25.4\%), low monthly income (41.2\%), and not married. However, in this study, there was no significant relationship between gender and the severity of depression symptoms in CKD patients on regular dialysis. 
This study has several weaknesses, one of which is that this study only focuses on measuring the magnesium levels of CKD patients on regular hemodialysis. Meanwhile, serum electrolyte levels such as sodium, potassium and chloride were not measured and statistically analyzed in this study. In addition, measuring magnesium levels is only done once, which is before the hemodialysis procedure. Therefore, it is not possible to know the changes in magnesium levels before and after hemodialysis and whether it has a significant effect on depression symptoms in CKD patients undergoing hemodialysis.

Another drawback of this study was that there was no analysis of the relationship between the length of hemodialysis and serum magnesium levels. In fact, the length of time undergoing hemodialysis can be one of the factors that influence the severity of depression complaints experienced by patients. Therefore, further research is needed to examine the relationship between the duration of hemodialysis and the severity of depression complaints as measured by the BDI II score.

\section{CONCLUSION}

Based on the research results that have been described in this study, the conclusions that can be drawn include: (1) there is a significant correlation between serum Magnesium levels and BDI II score with a weak correlation strength level $(\mathrm{r}=$ 0.35), (2) The BDI II score in regular hemodialysis patients was found to be in a score of 4-15 with a median score of 7 , and (3) Magnesium levels in regular hemodialysis patients are in the range 1.78 $3.75 \mathrm{mg} / \mathrm{dl}$, with a median of 2.9 .

Acknowledgement: None

Conflict of Interest: None

Source of Funding: None

Ethical Approval: Approved

\section{REFERENCES}

1. Kilzieh N, Rastam S, Maziak W, Ward K. Comorbidity of Depression with Chronic Diseases: A Population-Based Study in Aleppo, Syria. The International Journal of Psychiatry in Medicine. 2008;38(2):169-184.

2. Schmidt RJ and Holley JL. Psychiatric Illness in Dialysis Patients. UpToDate literature review version 17.3. 2009.

3. Flavio Teles, Milma Pires de Melo Vega Figueiredo Dourado de Azevedo. Depression in hemodialysis patients: the role of dialysis shift. Journal Clinics. 2013:198-202.

4. Ginieri-Coccossis M, Theofilou P, Synodinou C, Tomaras V, Soldatos C. Quality of life, mental health and health beliefs in haemodialysis and peritoneal dialysis patients: Investigating differences in early and later years of current treatment. BMC Nephrology. 2008;9(1).

5. Beck AT, Steer RA, Brown GK. BDI: Fast Screen for medical patients manual. San Antonio (TX): The Psychological Corporation. 2000:34

6. Serefko A, Szopa A, Wlaź P, Nowak G, Radziwoń-Zaleska M, Skalski M et al. Magnesium in depression. Pharmacological Reports. 2013;65(3): 547-554.

7. Nechifor M. Magnesium in major depression. Magnesium Research. 2009; 22(3):163S-166S.

8. Widmer J, Stella N, Raffin Y, Bovier P, Gaillard JM, Hilleret H, Tissot R. Blood magnesium, potassium, sodium, calcium and cortisol in drug-free depressed patients. Magnes Res. 1993 Mar;6(1): 33-41.

9. Imada $\mathrm{Y}$, Yoshioka S, Ueda $\mathrm{T}$, Katayama S, Kuno Y, Kawahara R. Relationships between serum magnesium levels and clinical background factors in patients with mood disorders. Psychiatry and Clinical Neurosciences. 2002;56(5):509-514.

10. Kaplan, H.I., Saddock, B.J, and Grebb, J.A. Sinopsis Psikiatri: Ilmu 
Miftahul Ihsan et.al. The correlation between beck depression inventory II (BDI II) score with serum magnesium levels in patients on regular hemodialysis.

Pengetahuan Perilaku Psikiatri Klinis Jilid I. Tangerang: Bina Rupa Aksara. 2010.

11. Iosifescu D, Bolo N, Nierenberg A, Jensen J, Fava M, Renshaw P. Brain Bioenergetics and Response to Triiodothyronine Augmentation in Major Depressive Disorder. Biological Psychiatry. 2008;63(12):1127-1134.
How to cite this article: Ihsan M, Lubis WH, Nasution AT. The correlation between beck depression inventory II (BDI II) score with serum magnesium levels in patients on regular hemodialysis. International Journal of Research and Review. 2021; 8(7): 176184. DOI: https://doi.org/10.52403/ijrr. 20210724

$* * * * * *$ 